\title{
Prehospital Delay and Stroke-related Symptoms
}

\author{
Tomoko Yanagida, Shigeru Fujimoto, Takuya Inoue and Satoshi Suzuki
}

\begin{abstract}
Objective Prehospital delay is the major cause of treatment delay in stroke. This study was conducted to clarify the contribution of specific stroke-related symptoms to prehospital delay.

Methods A consecutive series of 469 patients hospitalized within 2 weeks of stroke onset was collected. In this study, prehospital delay was defined as the time interval from recognition of stroke-related symptoms to hospital arrival. The prevalence of each symptom or sign and prehospital delay were analyzed.

Results Weakness of the lower limb (43.5\%) was the most common symptom followed by weakness of the upper limb (37.1\%) and dysarthria (31.6\%). Conversely, the most common sign was weakness of the upper limb (54.1\%) followed by dysarthria (53.3\%), weakness of the lower limb (53.1\%), and sensory disturbance $(39.0 \%)$. The presence of confusion/decreased level of consciousness $(\mathrm{p}<0.001)$, aphasia $(\mathrm{p}<0.001)$, headache $(\mathrm{p}=0.017)$, and nausea/vomiting $(\mathrm{p}=0.035)$ were associated with earlier hospital visitation compared with the absence of these symptoms in univariate analyses. Conversely, the presence of sensory disturbance ( $\mathrm{p}=$ $0.0017)$ and vertigo/dizziness $(\mathrm{p}=0.044)$ were associated with a significant delay in hospital visitation compared with the absence of these symptoms. There was a discrepancy in the prevalence between symptoms recognized by the patients or bystanders and signs diagnosed by the physicians.

Conclusion There was a significant overall correlation between prehospital delay and the National Institute of Health Stroke Scale scores. Public education is therefore necessary to encourage early hospital visitation even with the appearance of mild symptoms.
\end{abstract}

Key words: acute stroke, bystander, prehospital delay, sign, symptom

(Intern Med 54: 171-177, 2015)

(DOI: 10.2169/internalmedicine.54.2684)

\section{Introduction}

Time is a critical factor in treating acute stroke and transient ischemic attack (TIA), whether as thrombolytic therapy for ischemic stroke or blood pressure control for hemorrhagic stroke $(1,2)$. Prehospital delay continues to constitute the largest portion of delay time from the onset of disease to starting treatment in acute stroke $(3,4)$. Many efforts have been made to analyze the factors which affect prehospital delay in acute stroke patients (5-11). It has been previously reported that stroke-related symptoms may contribute to prehospital delay; however, the extent of this contribution differs for each symptom (12). Stroke-related symptoms have been analyzed based on signs which were diagnosed by the physicians, not based on symptoms recognized by the patients or bystanders. It is very important to know the preva- lence of both the patients or bystanders recognizing the symptoms and of the physicians recognizing the signs. The next step is to clarify the relation between each symptom and prehospital delay. Prehospital delay consists of two different elements; the first is the delay from the onset of disease to the recognition of the symptoms, and the second is the delay from the recognition of symptoms to hospital arrival. We herein focused on the latter to investigate the response of the patients or bystanders to the stroke-related symptoms. Prehospital delay was defined as the time interval from the recognition of the symptoms by the patients or bystanders to the hospital arrival. We investigated and compared the prevalence of each symptom and sign recognized by the patients or bystanders and the physicians. Prehospital delay, the National Institutes of Health Stroke Scale (NIHSS) scores, and emergency medical service (EMS) use were compared between the presence and absence of each 
symptom to understand which symptoms were associated with an earlier hospital visitation, which symptoms tended to be severe, and which symptoms prompted EMS use. Prehospital delay and the NIHSS score were examined to clarify whether the severity of the disease affected prehospital delay with each symptom.

\section{Materials and Methods}

\section{Study design and setting}

A database was constructed for acute cerebrovascular disease (CVD) patients at our institution. Patients were included if they visited the emergency department or the outpatient department no later than 2 weeks after the onset of stroke or TIA and were then admitted to our hospital. There were no exclusion criteria and all patients who met the criteria were registered to the database. A consecutive series of 469 patients was registered in this database from May 2007 to March 2009. A neurological examination was performed and recorded at the emergency room or the outpatient department by board certified stroke specialists or a board certified neurosurgeon for each patient. Additionally, diagnoses of acute stroke or TIA were made by board certified stroke specialists or a board certified neurosurgeon.

Steel Memorial Yawata Hospital, which is affiliated with the Kyushu University Hospital, is a core hospital in the western part of Kitakyushu, a city with a population of 0.97 million. Steel Memorial Yawata Hospital is a training hospital accredited by The Japan Stroke Society and The Japan Neurosurgical Society.

EMS is provided by the fire department of each regional government and is not charged to the patient in Japan. People can directly contact the fire department by calling 119 . If the request is deemed appropriate, an ambulance is dispatched immediately.

\section{Data collection and processing}

Data were collected during face-to-face interviews with research assistants using a structured interview guide developed by the investigators. These research assistants were trained by the authors periodically. The patients or bystanders were interviewed about whether they noticed confusion/ decreased level of consciousness, speech disturbance, facial weakness, weakness of the upper limb, weakness of the lower limb, sensory disturbance, visual problem, headache, nausea/vomiting, and vertigo/dizziness. Speech problems were divided into aphasia and dysarthria. Aphasia was defined as the inability of the patients to adequately verbalize their thoughts, idiosyncratic or nonsensical sentences, or poor verbal comprehension without a deterioration of consciousness. All symptoms which they recognized were recorded and multiple answers were allowed. Onset time, the time when the patients or bystanders recognized the symptoms, and the time of the hospital visit were recorded. If the onset time was unclear, it was defined as the last time when the patients were confirmed to be doing well. Prehospital delay was defined as the time interval from the recognition of symptoms by the patients or bystanders to hospital arrival. Data were entered into a spreadsheet for statistical analyses.

\section{Recognition of signs by the patients or bystanders}

The recognition of signs by the patients or bystanders was calculated and expressed as the following: (number of patients who had each sign recognized by the patient or bystanders) / (number of patients who had each sign) $\times 100$ (\%).

\section{Statistical analysis}

Descriptive statistics include the median and interquartile range (IQR). An analysis was performed using JMP 10 (SAS Institute, Cary, USA) and MedCalc ver. 12.3.0.0 (MedCalc Software bvba, Ostend, Belgium). Wilcoxon's non-parametric analyses were used to test differences in prehospital delay and the NIHSS score with the presence of each symptom. Spearman's rank correlation was utilized to examine the correlation between prehospital delay and the NIHSS score at the first neurological examination. Fisher's exact test was used to compare EMS use between the absence and presence of each symptom. p values less than 0.05 were considered to be significant.

\section{Results}

\section{Characteristics of the study subjects}

A total of 469 patients were enrolled in the present study: 222 were women $(47.3 \%), 437$ patients $(93.2 \%)$ resided at home, 90 patients $(19.4 \%)$ resided alone, 390 patients (83.2\%) were independent in their daily activity (modified Rankin scale $\leq 2), 251$ patients $(53.5 \%)$ used EMS, and 36 patients $(7.7 \%)$ had knowledge of alteplase (Table 1). Thirty patients out of 369 patients (three were excluded due to missing data) with ischemic stroke or TIA had knowledge of alteplase. Stroke-related symptoms were recognized by the patients themselves for 288 patients $(61.4 \%)$, by the spouse for 46 patients $(9.8 \%)$, by other family members or relatives for 55 patients $(12.2 \%)$, and by others for 78 patients $(16.6 \%)$. Stroke subtypes were ischemic stroke in 342 patients $(72.9 \%)$, intracerebral hemorrhage in 74 patients (15.8\%), subarachnoid hemorrhage in 23 patients (4.9\%), and TIA in 30 patients $(6.4 \%)$. Intravenous recombinant tissue plasminogen activator (rt-PA) injection was performed in 36 patients (9.7\% of ischemic stroke and TIA patients). No TIA patients received intravenous rt-PA therapy. The median time of prehospital delay was 6.6 hours (IQR, 1.2-43.6 hours). The median time of prehospital delay was 3.0 hours (IQR, 1.1-98.2 hours) in TIA patients and 6.7 hours (IQR, 1.2-43.0 hours) in stroke patients, respectively $(\mathrm{p}=0.80)$. The median NIHSS score in the first neurological examination was 4 (IQR, 1-8). The NIHSS score was 4 or lower in 255 
Table 1. Baseline Characteristics of Patients

\begin{tabular}{|c|c|}
\hline & $\begin{array}{c}\mathrm{N} \text { or median } \\
(\mathrm{IQR} \text { or } \%)\end{array}$ \\
\hline $\mathrm{N}$ of patients & 469 \\
\hline Age, y & $75(67-81)$ \\
\hline$\geq 75 y(\%)$ & $236(50.3)$ \\
\hline Women $(\%)$ & $222(47.3)$ \\
\hline Resides at home (\%) & $437(93.2)$ \\
\hline Resides alone (\%) & $90(19.4)$ \\
\hline Estimated NIHSS & $4(1-8)$ \\
\hline NIHSS $\geq 5(\%)$ & $214(45.6)$ \\
\hline Delay, hours & $6.6(1.2-43.6)$ \\
\hline Prestroke mRS & $0(0-1)$ \\
\hline $\mathrm{mRS} \leq 2(\%)$ & $390(83.2)$ \\
\hline Use of EMS (\%) & $251(53.5)$ \\
\hline Knowledge of rt-PA & 36 \\
\hline in whole patients $(\%)$ & $(7.7)$ \\
\hline in infarct or TIA patients (\%) & $(9.7)$ \\
\hline \multicolumn{2}{|l|}{ Who recognized symptoms? (\%) } \\
\hline Patient & $288(61.4)$ \\
\hline Spouse & $46(9.8)$ \\
\hline Other family member or relative & $55(12.2)$ \\
\hline Others & $78(16.6)$ \\
\hline \multicolumn{2}{|l|}{ Stroke type $(\%)$} \\
\hline $\mathrm{ICH}$ & $74(15.8)$ \\
\hline Infarct & $342(72.9)$ \\
\hline SAH & $23(4.9)$ \\
\hline TIA & $30(6.4)$ \\
\hline
\end{tabular}

Numbers are median and interquartile range (IQR).

NIHSS: National Institute of Health Stroke Scale, mRS: modified Rankin scale, EMS: emergency medical service, rt-PA: recombinant tissue plasminogen activator, ICH: intracerebral hemorrhage, SAH: subarachnoid hemorrhage, TIA: transient ischemic attack

patients $(54.4 \%)$. Table 1 shows the baseline characteristics of patients.

\section{Prevalence of each symptom or sign}

The prevalence of each symptom was as follows: confusion/decreased level of consciousness in 83 patients; dysarthria in 148 patients; aphasia in 23 patients; facial weakness in 25 patients; weakness of the upper limb in 174 patients; weakness of the lower limb in 204 patients; sensory disturbance in 63 patients; visual problem in 11 patients; headache in 52 patients; nausea/vomiting in 57 patients; and vertigo/dizziness in 57 patients (Fig. 1, 2).

The prevalence of each sign was as follows: confusion/decreased level of consciousness in 109 patients; dysarthria in 250 patients; aphasia in 72 patients; facial weakness in 167 patients; weakness of the upper limb in 254 patients; weakness of the lower limb in 249 patients; sensory disturbance in 183 patients; and visual problem in 33 patients. No signs were recognized during the physician's neurological examination in 54 patients (11.5\%, Fig. 1).

\section{Recognition of signs by the patients or bystanders}

Weakness of the lower limb $(81.9 \%)$, confusion/decreased level of consciousness $(75.5 \%)$, and weakness of the upper limb $(68.5 \%)$ were well recognized by the patients or bystanders (Fig. 2). Conversely, facial weakness (15.0\%), aphasia $(31.9 \%)$, visual problem $(33.3 \%)$, and sensory dis- turbance $(34.4 \%)$ were poorly recognized by the patients or bystanders.

\section{Prehospital delay in each symptom}

The presence of confusion/decreased level of consciousness $(p<0.001)$, aphasia $(p<0.001)$, headache $(p=0.017)$, and nausea/vomiting $(\mathrm{p}=0.035)$ were associated with earlier hospital visitation compared with the absence of these symptoms in the univariate analyses (Table 2, Fig. 3). Conversely, the presence of sensory disturbance $(\mathrm{p}=0.0017)$ and vertigo/ dizziness $(\mathrm{p}=0.044)$ were associated with a significant delay in hospital visitation compared with the absence of these symptoms.

\section{NIHSS scores in each symptom}

The NIHSS score was higher in the presence of confusion/decreased level of consciousness $(\mathrm{p}<0.001)$, aphasia $(\mathrm{p}=$ $0.0013)$, weakness of the upper limb $(\mathrm{p}<0.001)$, and weakness of the lower limb $(\mathrm{p}<0.001)$ compared with the absence of these symptoms in the univariate analyses (Table 2). Conversely, the NIHSS score was lower in the presence of dysarthria $(\mathrm{p}=0.036)$, sensory disturbance $(\mathrm{p}=0.0002)$, visual problem ( $\mathrm{p}=0.0066)$, nausea/vomiting $(\mathrm{p}=0.0054)$, and vertigo/dizziness $(\mathrm{p}<0.0001)$ compared with the absence of these symptoms.

\section{EMS use in each symptom}

The presence of confusion/decreased level of consciousness $(p<0.001)$, headache $(p=0.0053)$, and nausea/vomiting $(p<0.001)$ were associated with more frequent use of EMS compared with absence of these symptoms in the univariate analyses. Conversely, the presence of sensory disturbance $(\mathrm{p}=0.0002)$ and visual problem $(\mathrm{p}=0.028)$ were associated with less frequent use of EMS (Table 2).

\section{Correlations between prehospital delay and NIHSS score}

Rank correlation tests using Spearman's coefficient (rho) revealed that there were negative correlations between prehospital delay and the NIHSS score overall $(\mathrm{p}<0.001)$ and for confusion/decreased level of consciousness $(\mathrm{p}=0.027)$, dysarthria $(p<0.001)$, facial weakness $(p=0.013)$, weakness of the upper limb $(\mathrm{p}<0.001)$, weakness of the lower limb ( $\mathrm{p}$ $<0.001)$, and sensory disturbance $(\mathrm{p}=0.017)$ (Table 2).

\section{Discussion}

In this single center study, we tried to clarify the relationship between prehospital delay and symptoms recognized by the patients or bystanders during acute stroke. To our knowledge, this is the first report focused on the detailed symptoms recognized by the patients or bystanders. There are several reports concerning relationships between prehospital delay and symptoms; however, the symptoms in these reports are signs diagnosed by the physicians, not recognized by the patients or bystanders $(12,13)$. In our study, 


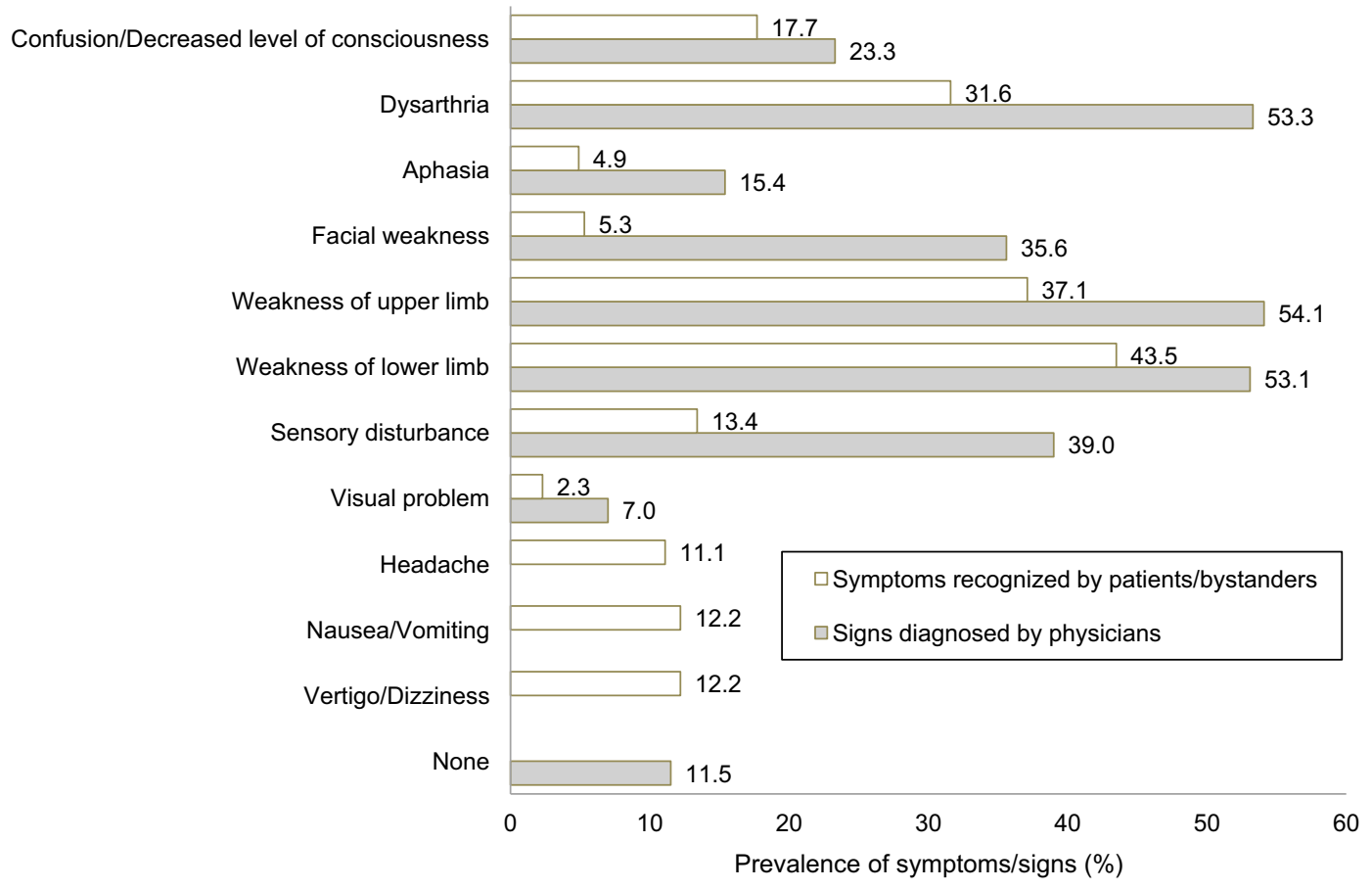

Figure 1. The prevalence of stroke-related symptoms and signs.

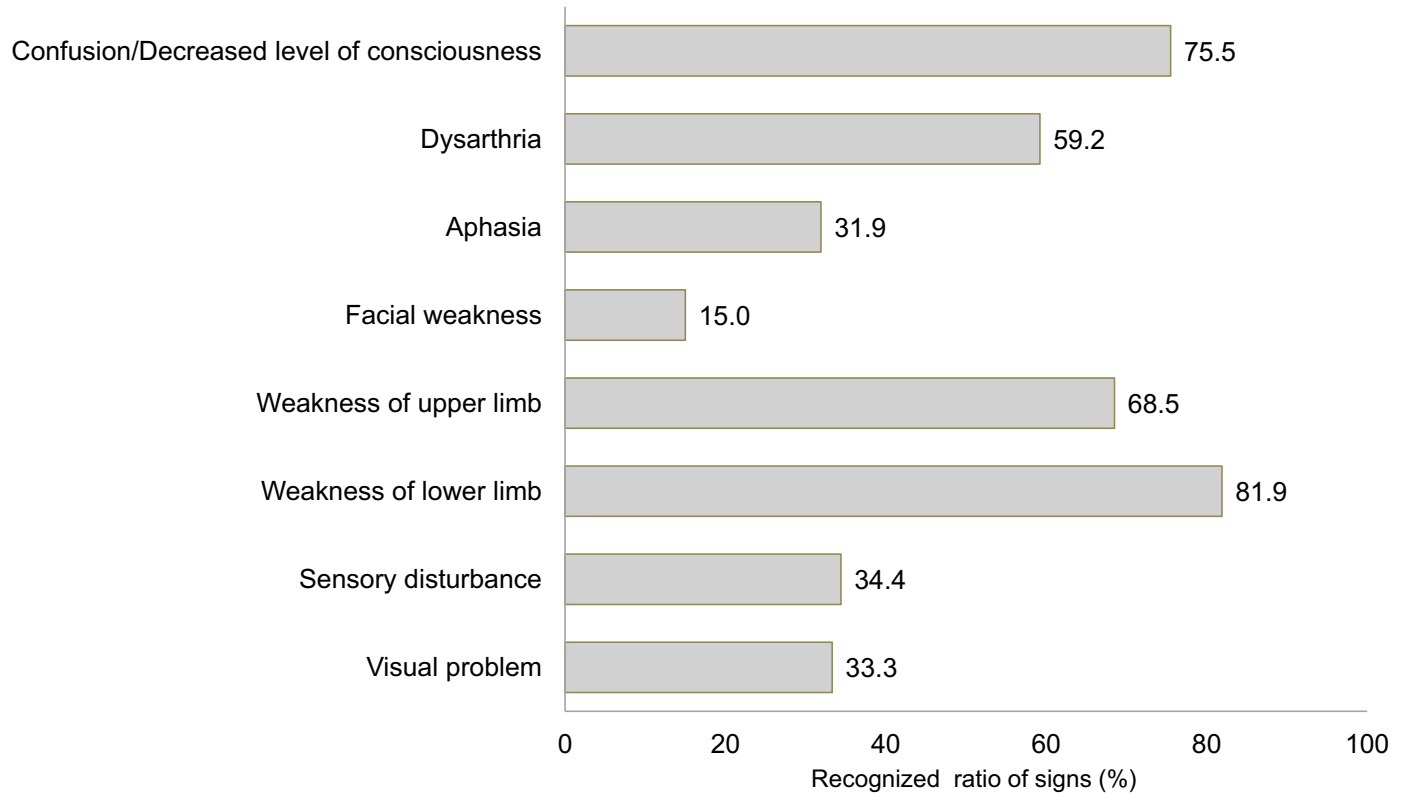

Figure 2. The recognized ratio of signs by the patients/bystanders.

only $15.0 \%$ of facial weakness and $31.9 \%$ of aphasia were recognized by the patients or bystanders. The general public seems to be much more sensitive to weakness of the upper or lower limb and dysarthria rather than facial weakness and aphasia. The presence of sensory disturbance $(\mathrm{p}=0.0017)$ and vertigo/dizziness $(\mathrm{p}=0.044)$ were associated with a significant delay in hospital visitation compared with the absence of these symptoms. The NIHSS score tended to be lower with these symptoms; therefore, public education to promote early hospital visitation, even with milder symptoms, is critical. Additionally, it is necessary to promote early hospital visitation even with transient symptoms.
Whether knowledge of stroke-related symptoms facilitates earlier hospital visitation still remains controversial. Fussman et al. suggested the lack of an association between stroke symptom knowledge and intent to use EMS (14). Conversely, Kim et al. suggested that knowledge of strokerelated symptoms decreased prehospital delay (10). It has been widely reported that use of EMS is intended to minimize prehospital delay. Our results are compatible with these previously reported data. Furthermore, knowledge of rt-PA means that the patients realized that stroke should be treated as early as possible. This type of information for the general public is quite important in reducing prehospital de- 


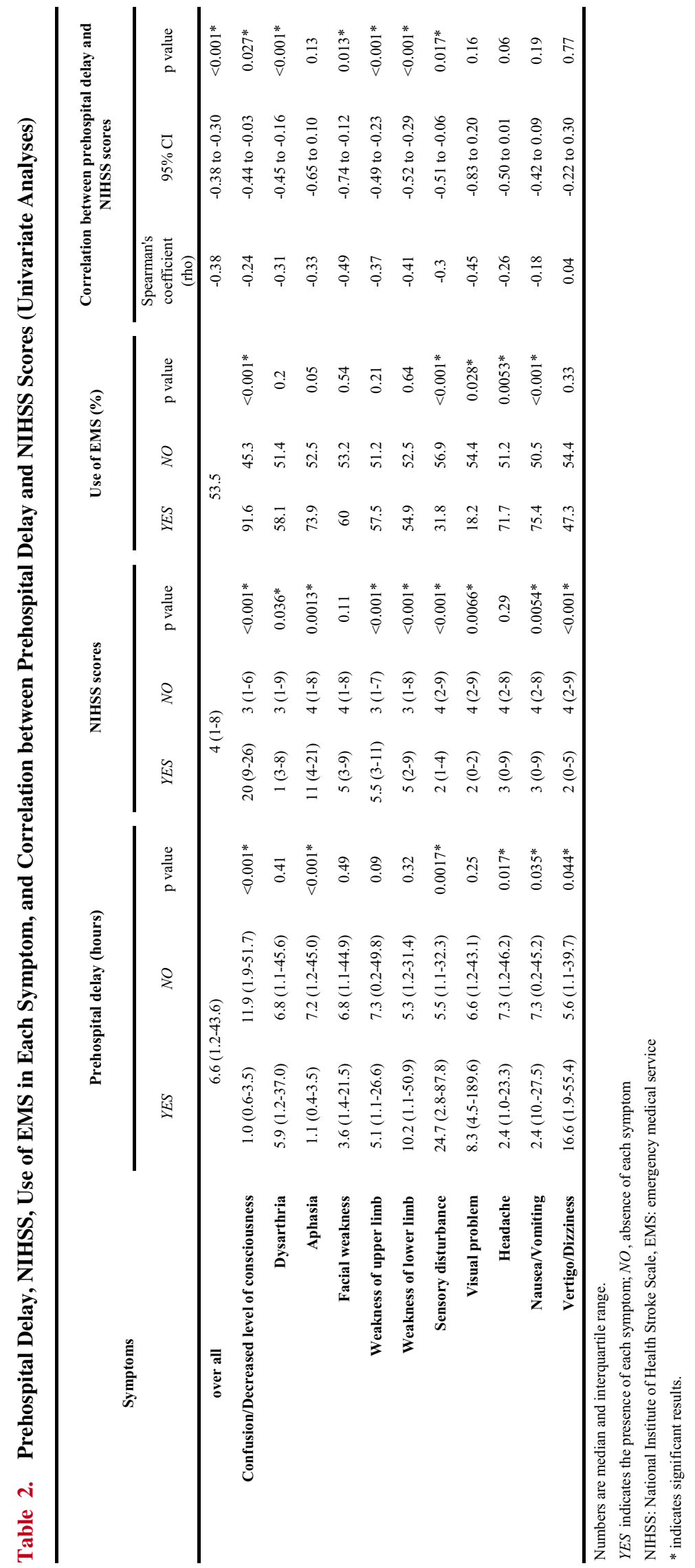




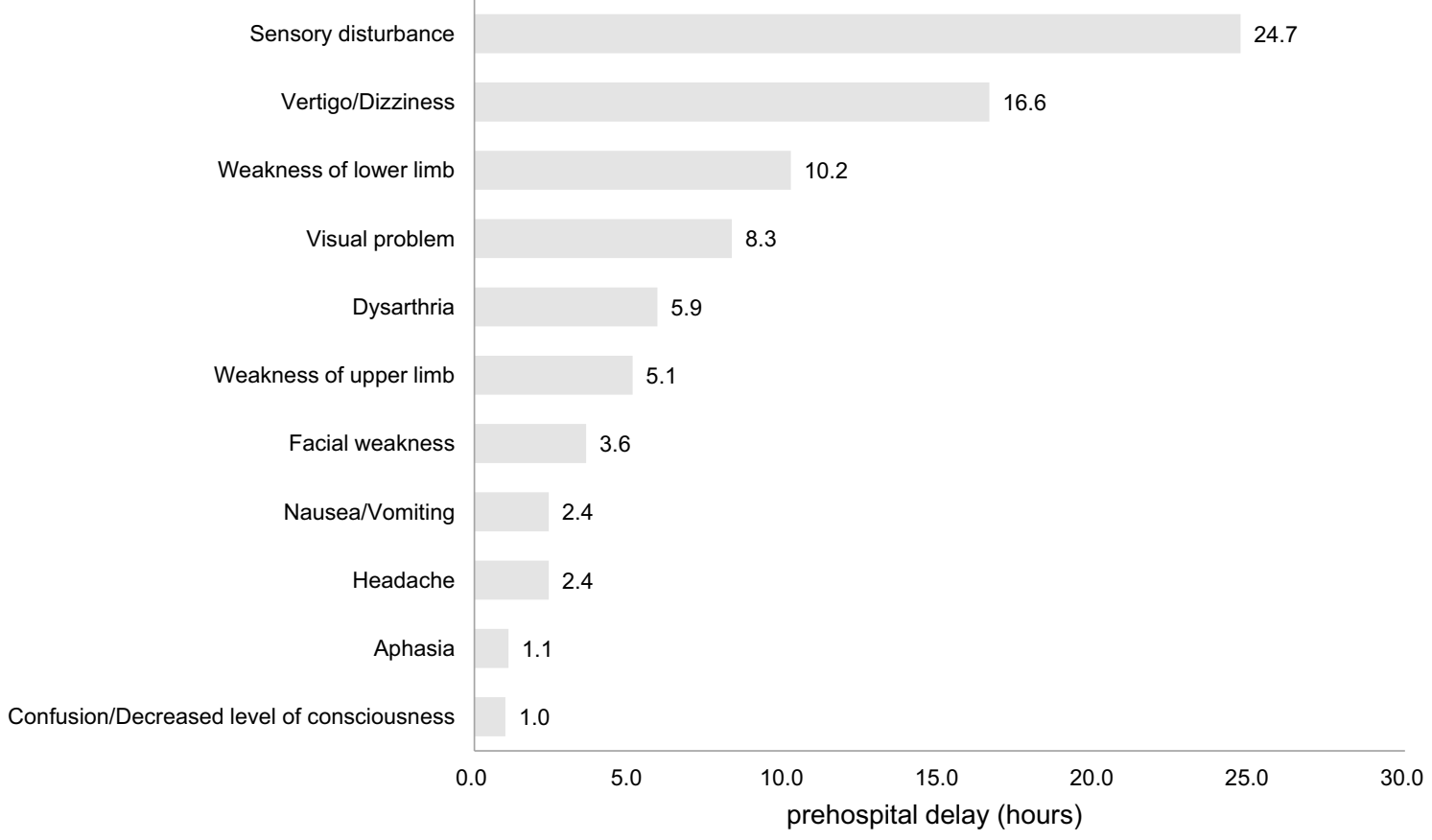

Figure 3. The median prehospital delay of each symptom.

lay.

Prehospital delay in our study was defined as the time interval from recognition of symptoms by the patients or bystanders to hospital arrival; therefore, the time interval from the onset of symptoms to the recognition of symptoms was not included. The median time from the onset of symptoms to hospital arrival was 12.7 hours (IQR, 2.1-46.6), which is longer than the median time of our prehospital delay (6.6 hours; IQR, 1.2-43.6). In a considerable number of cases, the onset time of stroke-related symptoms is not clear; therefore, the time interval from the onset to the recognition of symptoms may be ambiguous. This is one reason why we did not use this time interval. Furthermore, our aim was to determine the differences in response of the patients or bystanders to each symptom. This is the main reason why we defined prehospital delay as the time interval from the recognition of symptoms by the patients or bystanders to hospital arrival.

It has been reported that the severity of the symptoms affects prehospital delay, i.e., relatively mild symptoms cause longer prehospital delays (5). In our study, prehospital delay was negatively correlated with the NIHSS score overall (Spearman's coefficient $-0.38,95 \%$ CI -0.46 to -0.30 , p $<$ 0.001 ) and with confusion/decreased level of consciousness, dysarthria, facial weakness, weakness of the upper limb, weakness of the lower limb, and sensory disturbance. No correlation was seen between prehospital delay and aphasia, visual problem, headache, nausea/vomiting, or vertigo/dizziness. It has been reported that the NIHSS score is more weighted toward anterior-circulation stroke symptoms. This may be one reason why prehospital delay was not correlated with the NIHSS score for these symptoms (15). These results suggest that patients tend to visit the hospital earlier regardless of the severity of symptoms if the patients or bystanders recognized aphasia, headache, or nausea/vomiting. Aphasia may cause an earlier hospital visitation regardless of the severity of symptoms due to accompanying symptoms, such as paralysis of the dominant hand, agnosia, or apraxia. In the case of sensory aphasia, the bystanders may recognize a deterioration of the patient's consciousness level or decreased attention.

There are several limitations associated with our study. First, this represents the experience of a single center. As such, local factors may have influenced the results. The single study site may prohibit drawing inferences about other settings and populations. Second, the number of patients was not large enough to allow subgroup analyses. For example, visual problems were recognized only by 11 patients or bystanders $(2.3 \%)$; this was too small a sample to make a detailed analysis. Third, the presentation of each symptom was mostly in combination with other symptoms; therefore, it is very difficult to exclude the interference of each symptom even after using the multivariate analyses. However, we believed that it was worthwhile to analyze prehospital delay purely from the symptoms recognized by the patients/bystanders. A multi-site study or enrollment of a larger number of patients is therefore warranted.

In conclusion, the presence of confusion/decreased level of consciousness $(p<0.001)$, aphasia $(p<0.001)$, headache ( $\mathrm{p}=0.017)$, and nausea/vomiting $(\mathrm{p}=0.035)$ were associated with earlier hospital visitation compared with an absence of these symptoms in the univariate analyses. Conversely, the presence of sensory disturbance $(\mathrm{p}=0.0017)$ and vertigo/dizziness $(p=0.044)$ were associated with a significant delay in hospital visitation compared with an absence of these symptoms. We recommend the promotion of early hospital visita- 
tion for patients with acute stroke and TIA patients even with the appearance of mild symptoms.

The authors state that they have no Conflict of Interest (COI).

\section{Acknowledgement}

The authors thank the staff of their stroke center for help in performing this study.

\section{References}

1. Hacke W, Kaste M, Bluhmki E, et al. Thrombolysis with alteplase 3 to 4.5 hours after acute ischemic stroke. N Engl J Med 359: 1317-1329, 2008.

2. Anderson CS, Huang Y, Wang JG, et al. Intensive blood pressure reduction in acute cerebral hemorrhage trial (INTERACT): a randomized pilot trial. Lancet Neurol 7: 391-399, 2008.

3. Evenson KR, Foraker R, Morris DL, Rosamond WD. A comprehensive review of prehospital and in-hospital delay times in acute stroke care. Int J Stroke 4: 187-199, 2009.

4. Bouckaert M, Lemmens R, Thijs V. Reducing prehospital delay in acute stroke. Nat Rev Neurol 5: 477-483, 2009.

5. Jorgensen HS, Nakayama H, Reith J, Raaschou HO, Olsen TS. Factors delaying hospital admission in acute stroke: the Copenhagen Stroke Study. Neurology 47: 383-387, 1996.

6. Evenson KR, Rosamond WD, Morris DL. Prehospital and inhospital delays in acute stroke care. Neuroepidemiology 20: 65-
76, 2001.

7. Chang KC, Tseng MC, Tan TY. Prehospital delay after acute stroke in Kaohsiung, Taiwan. Stroke 35: 700-704, 2004.

8. Teuschl Y, Brainin M. Stroke education: discrepancies among factors influencing prehospital delay and stroke knowledge. Int $\mathbf{J}$ Stroke 5: 187-208, 2010.

9. Hong ES, Kim SH, Kim WY, Ahn R, Hong JS. Factors associated with prehospital delay in acute stroke. Emerg Med J 28: 790-793, 2011.

10. Kim YS, Park SS, Bae HJ, et al. Stroke awareness decreases prehospital delay after acute ischemic stroke in Korea. BMC Neurol 11: 2, 2011.

11. Jin H, Zhu S, Wei JW, et al; China QUEST (Quality Evaluation of Stroke Care and Treatment) Investigators. Factors associated with prehospital delays in the presentation of acute stroke in urban China. Stroke 43: 363-370, 2012.

12. Kleindorfer D, Lindsell CJ, Moomaw CJ, et al. Which stroke symptoms prompt a 911 call? A population-based study. Am J Emer Med 28: 607-612, 2010.

13. Tanaka Y, Nakajima M, Hirano T, Uchino M. Factors influencing pre-hospital delay after ischemic stroke and transient ischemic attack. Inter Med 48: 1739-1744, 2009.

14. Fussman C, Raffery AP, Lyon-Callo S, Morgenstern LB, Reeves MJ. Lack of association between stroke symptom knowledge and intent to call 911: A population-based survey. Stroke 41: 15011507, 2010.

15. Sato S, Toyoda K, Uehara T, et al. Baseline NIH Stroke Scale Score predicting outcome in anterior and posterior circulation strokes. Neurology 70: 2371-2377, 2008.

(C) 2015 The Japanese Society of Internal Medicine http://www.naika.or.jp/imonline/index.html 